The AstrophysiCAL Journal, 504:889-899, 1998 September 10

(C) 1998. The American Astronomical Society. All rights reserved. Printed in U.S.A.

\title{
STATISTICAL PROPERTIES OF LINE CENTROID VELOCITY INCREMENTS IN THE $\rho$ OPHIUCHI CLOUD
}

\author{
D. C. Lis, Jocelyn Keene, Y. Li, AND T. G. Phillips \\ California Institute of Technology, Downs Laboratory of Physics 320-47, Pasadena, CA 91125
}

AND

J. PETY

CNRS URA 336, Ecole Normale Supérieure, 24 rue Lhomond, 75005 Paris, France Received 1997 September 15 ; accepted 1998 April 15

\begin{abstract}
We present a comparison of histograms of CO (2-1) line centroid velocity increments in the $\rho$ Ophiuchi molecular cloud with those computed for spectra synthesized from a three-dimensional, compressible, but non-star-forming and nongravitating, hydrodynamic simulation. Histograms of centroid velocity increments in the $\rho$ Oph cloud clearly show non-Gaussian wings similar to those found in histograms of velocity increments and derivatives in experimental studies of laboratory and atmospheric flows, as well as numerical simulations of turbulence. The magnitude of these wings increases monotonically with decreasing separation, down to the angular resolution of the data. This behavior is consistent with that found in the phase of the simulation that has most of the properties of incompressible turbulence. The time evolution of the magnitude of the non-Gaussian wings in the histograms of centroid velocity increments in the simulation is consistent with the evolution of the vorticity in the flow. We cannot exclude, however, the possibility that the wings are associated with the shock interaction regions. Moreover, the effects of shocks may be more important in an active star-forming region like the $\rho$ Oph cloud than in the simulation; however, being able to identify shock interaction regions in the interstellar medium is also important, since numerical simulations show that vorticity is generated in shock interactions.
\end{abstract}

Subject headings: hydrodynamics - ISM: clouds — ISM: individual ( $\rho$ Ophiuchi Cloud $)-$ ISM: kinematics and dynamics — turbulence

\section{INTRODUCTION}

Early spectroscopic observations of interstellar lines of $\mathrm{H}$ I, $\mathrm{OH}$, and $\mathrm{CO}$ have revealed that observed line widths (or velocity dispersions) in interstellar clouds are larger than thermal line widths expected for these low-temperature regions (see, e.g., Myers 1997 and references therein). These large line widths are indicative of supersonic motions of the gas; however, the exact nature of these motions is still a subject of controversy. Proposed explanations generally involve turbulent motions of the gas, be it hydrodynamic or magneto-hydrodynamic (see, e.g., Scalo 1987; Falgarone 1997).

The idea that the gas motions in interstellar clouds are of turbulent nature was first proposed by Zuckerman \& Evans (1974). As discussed by Falgarone (1997), gas motions in interstellar clouds exhibit many features of incompressible turbulence. One of them is the size-line width relation first discussed by Larson (1981). This power-law scaling with an exponent of $\sim 0.3-0.5$, found in the velocity field of molecular clouds, is reminiscent of the Kolmogorov cascade in incompressible turbulence. Another possible signpost of turbulence is the fractal structure of the intensity contours in molecular clouds (see, e.g., Scalo 1990; Falgarone, Phillips, \& Walker 1991). In both cases alternative explanations have been proposed. An explanation for the size-line width relation observed from cloud to cloud in a given molecular line has been proposed based on virial equilibrium and the relatively small variation of the observed column density between clouds of different sizes (Myers 1997). Explanations for the observed scaling laws have also been given in terms of the framework of magnetically supported cloud models (Scalo 1987). Alternative explanations for the fractal cloud geometry based on collisional fragmentation or gravitational clustering and virial balance have also been proposed (Nozakura 1990; Pfenniger \& Combes 1994).

One of the tools employed in studies of gas motions in the interstellar medium is the analysis of shapes of molecular line profiles. Falgarone \& Phillips (1990) argued that the non-Gaussian CO line wings in inactive regions without associated star formation activity represent a direct observational signature of the turbulent nature of the gas flow within molecular clouds and of the existence of regions of intermittent turbulent activity. In a subsequent study, Falgarone et al. (1994) showed that line profiles synthesized from a three-dimensional turbulent, compressible, but nonstar-forming and nongravitating simulation (Porter, Pouquet, \& Woodward 1994) are in fact statistically similar to the $\mathrm{CO}$ line profiles observed in quiescent molecular clouds. Dubinski, Narayan, \& Phillips (1994), however, showed that non-Gaussian line profiles can also be produced from a random velocity field with a Kolmogorov power spectrum.

It is known from numerical simulations and atmospheric and laboratory measurements (see, e.g., Anselmet et al. 1984; Gagne 1987; Vincent \& Meneguzzi 1991) that intermittency of turbulence manifests itself through nonGaussian wings in probability density functions (PDFs or histograms) of velocity increments and derivatives. These kinds of measurements are not directly possible in the case of the interstellar medium where one has information integrated over a line-of-sight column defined by the passage of the telescope beam through the medium. However, in a recent study (Lis et al. 1996) motivated by observational work of Miesch \& Scalo (1995), we showed that histograms 
of the centroid velocity increments (or differences between line centroid velocities at positions separated by a given distance) for sections of the simulation data cube corresponding to the ISM columns also show non-Gaussian wings similar to those found in experiments and numerical simulations of incompressible turbulence. Owing to line-ofsight averaging, the wings seen in PDFs of centroid velocity increments are not as pronounced as those in PDFs of velocity increments calculated over the whole data cube. Nevertheless, the effect is clearly present. We also demonstrated that the lines of sight contributing to the nonGaussian wings of the PDFs of centroid velocity increments trace a filamentary structure, which follows the distribution of the two vorticity components involving cross-derivatives of the line-of-sight component of the velocity field. This suggests that the wings are a manifestation of the turbulent nature of the flow.

The method presented by Lis et al. (1996) provides a useful new tool for studying the effects of intermittency of turbulence on physics and chemistry of the interstellar medium. In the present paper we apply this method to a large-scale map of the CO (2-1) emission from the $\rho$ Ophiuchi molecular cloud. It is true that this region with active low-mass star formation may not be ideal for a study of turbulence, since the shocks associated with embedded young stellar objects can have their signatures in the velocity field. But on the other hand, the energy injected into the cloud by the embedded sources may also play an important role in generating the turbulent cascade. A statistical comparison of the velocity field in both active and quiescent regions is thus required. (Observations of a truly quiescent region are in progress.) In addition, we extend our analysis to two additional epochs of the simulation of Porter et al. (1994) to further investigate the origin of the non-Gaussian wings in the PDFs of centroid velocity increments in the simulation.

\section{OBSERVATIONS}

Observations of the CO (2-1) emission from the $\rho$ Oph molecular cloud presented here were carried out between 1994 July and 1995 March using the $10.4 \mathrm{~m}$ Leighton telescope of the Caltech Submillimeter Observatory (CSO) on Mauna Kea, Hawaii. The data set consists of 18 partially overlapping on-the-fly (OTF) maps. The relative calibration between the various maps was determined from the overlap regions, and the maps were scaled by the corresponding intensity ratios. The final map thus has a uniform calibration. Since not all the spectra were taken on the same regular grid in the equatorial coordinate system, a final regular grid map with $15^{\prime \prime}$ spacing was produced by averaging spectra falling with $8^{\prime \prime}$ radius from a given grid point [the CSO beam at the frequency of the CO (2-1) line is $\left.\sim 31^{\prime \prime}\right]$. This final resampled map consists of 8970 spectra taken over an area of $\sim 30^{\prime} \times 27^{\prime}$. A gray-scale image of the integrated line intensity between -4 and $12 \mathrm{~km} \mathrm{~s}^{-1}$ is shown in Figure 1, and velocity channel maps are shown in Figure 2. In spite of the fact that the data were generally taken under less than optimum weather conditions, the resulting signal-to-noise ratio for the integrated intensity is quite high (12-280). In the subsequent analysis we use a 15"

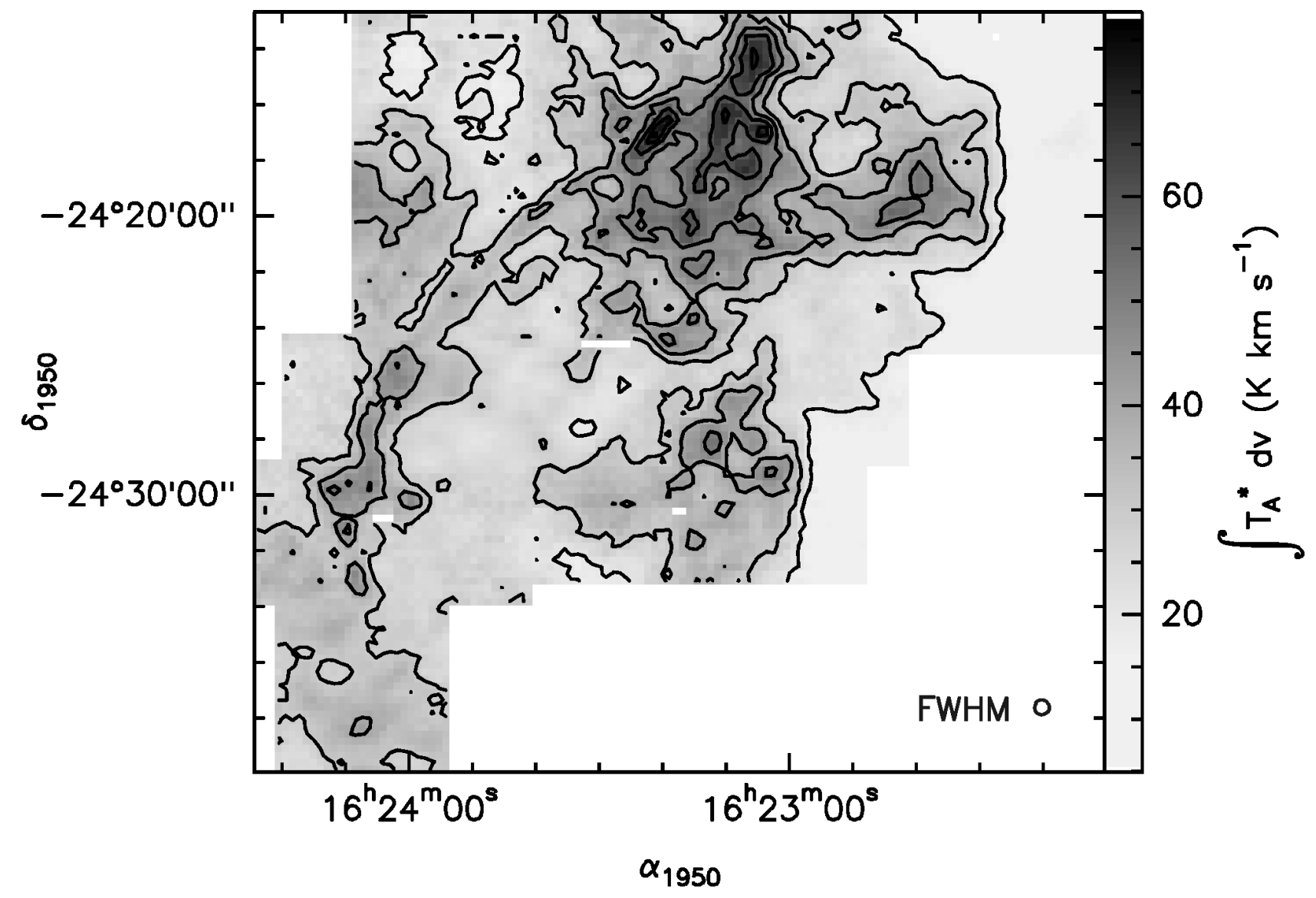

FIG. 1.-Gray-scale map of the $\mathrm{CO}(2-1)$ integrated intensity between -4 and $12 \mathrm{~km} \mathrm{~s}^{-1}$ in the $\rho$ Oph cloud. Contour levels are from 20 to 70 by $10 \mathrm{~K} \mathrm{~km}$ $\mathrm{s}^{-1}$. The intensity has not been corrected for the main-beam efficiency, which is $\sim 75 \%$ at the frequency of the CO (2-1) line. 


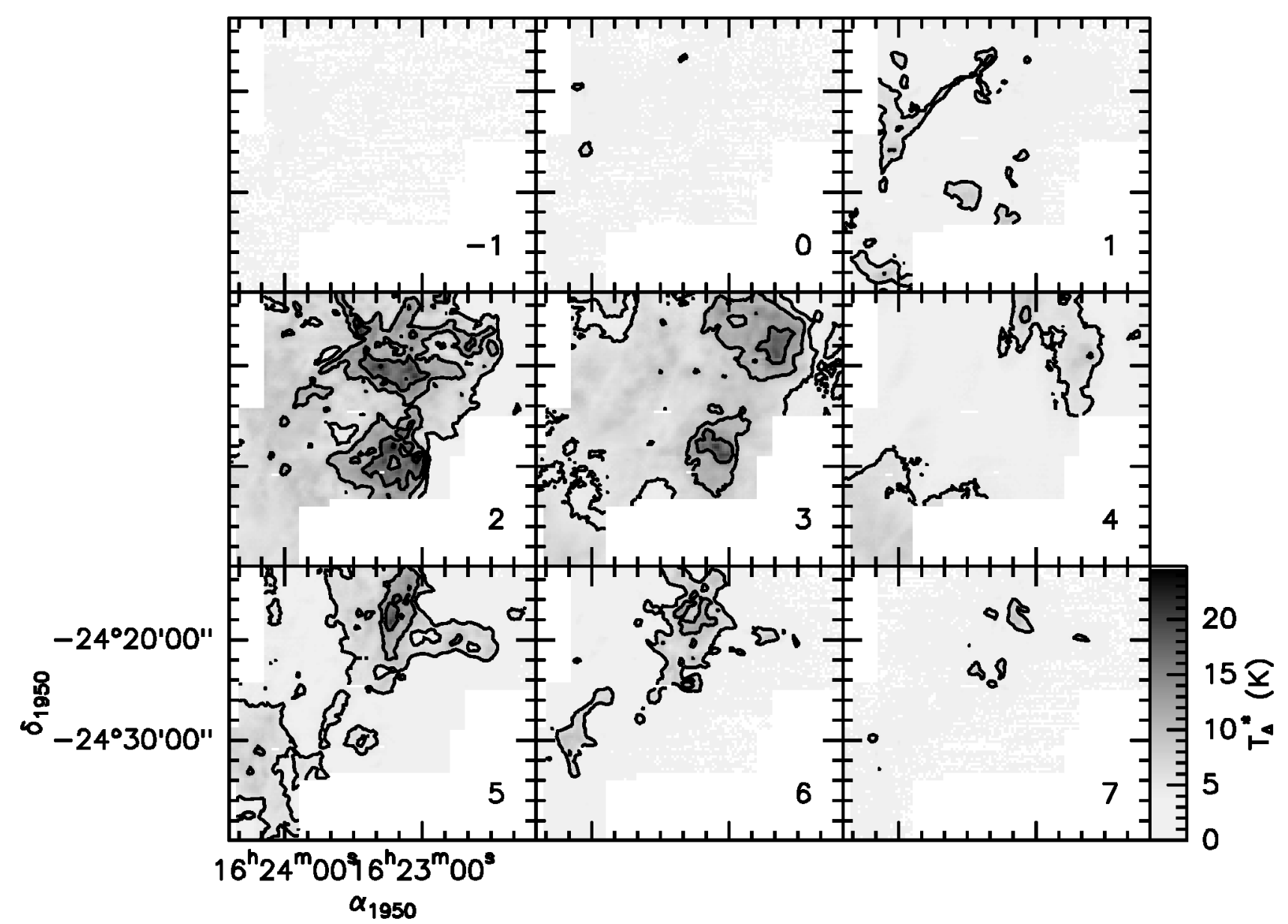

FIG. 2. - Channel maps of the $\mathrm{CO}(2-1)$ emission in the $\rho$ Oph cloud. Center velocities are written in the bottom right-hand corner of each panel. Contour levels are $5,10,15$, and $22 \mathrm{~K}$.

pixel as a length unit for the $x$ - and $y$-axes. The size of our map is $122 \times 109$ pixels.

\section{DISCUSSION}

The molecular cloud near $\rho$ Oph is one of the closest clouds, ranging in distance from about 80 to $200 \mathrm{pc}$. This thickness is thought to be real (de Geus, de Zeeuw, \& Lub 1989; de Geus \& Burton 1991). The cloud is brightly illuminated by the Sco OB 2 association, the closest such association to the Sun. The center of the Upper Scorpius subgroup lies to the west of the $\rho$ Oph cloud, and its stars lie at the same distance or beyond the cloud. Some of the stars appear to lie within the cloud.

In addition, the $\rho$ Oph cloud is a site of ongoing lowmass star formation. This cloud has been the subject of many near-infrared surveys over the years (see, e.g., Grasdalen, Strom, \& Strom 1973; Elias 1978; Wilking \& Lada 1983; Greene \& Young 1992; Barsony et al. 1997). Most of these surveys have been of limited sensitivity or of small areas. In order to confirm the membership of a source in the embedded infrared stellar cluster, it is good to have data in a variety of wavelength bands: optical; near-, mid-, and far-infrared; radio; and X-ray. Only of order 100 sources have been identified so far as being probable $\rho$ Oph cloud members (Wilking \& Lada 1983; Strom, Kepner, \& Strom 1995; Casanova et al. 1995). The rest simply do not have enough information available for them. The most recent near-infrared survey, Barsony et al. (1997), is a highsensitivity survey of a large area $\left(\sim 1 \mathrm{deg}^{2}\right)$, which catalogs 4495 sources. Analysis of this data set in terms of cloud membership has not been completed. Probable $\rho$ Oph cloud members are identified in Figure 3. The symbols indicate near-infrared, X-ray, and radio sources and optical emission-line stars. Many sources have been detected in more than one band. The sources are seen to be concentrated in two regions near the dense molecular cores, called $\rho$ Oph A and C. This is partly due to the fact that these areas are among the most thoroughly surveyed, but nevertheless it appears to be a real effect.

The most luminous of the stars in the region that we have mapped are HD $147889\left(\mathrm{~B} 2 \mathrm{~V} ; 5500 L_{\odot} ; 16^{\mathrm{h}} 22^{\mathrm{m}} 22^{\mathrm{s}} .8\right.$, $\left.-24^{\circ} 21^{\prime} 07^{\prime \prime}\right)$, S1 (B3 V; $\left.1500 L_{\odot} ; 16^{\mathrm{h}} 23^{\mathrm{m}} 32^{\mathrm{s}} .8,-24^{\circ} 16^{\prime} 44^{\prime \prime}\right)$ and SR 3 (B9 V; $\left.125 L_{\odot} ; 16^{\mathrm{h}} 23^{\mathrm{m}} 07^{\mathrm{s}} \mathrm{s},-24^{\circ} 27^{\prime} 26^{\prime \prime}\right)$. There are several sources with luminosities in the $10-50 L_{\odot}$ range, but most of them have luminosities of order $1 L_{\odot}$ or less (Wilking, Lada, \& Young 1989).

Stars can input energy into molecular clouds in ways other than through optical radiation. Many of the embedded stars are X-ray sources (Montmerle et al. 1983; Casanova et al. 1995). Also, there are several outflow sources known. Among these is the source of the extremely long outflow seen in Figure 1-VLA $1623\left(16^{\mathrm{h}} 23^{\mathrm{m}} 25^{\mathrm{s}} 0\right.$, 

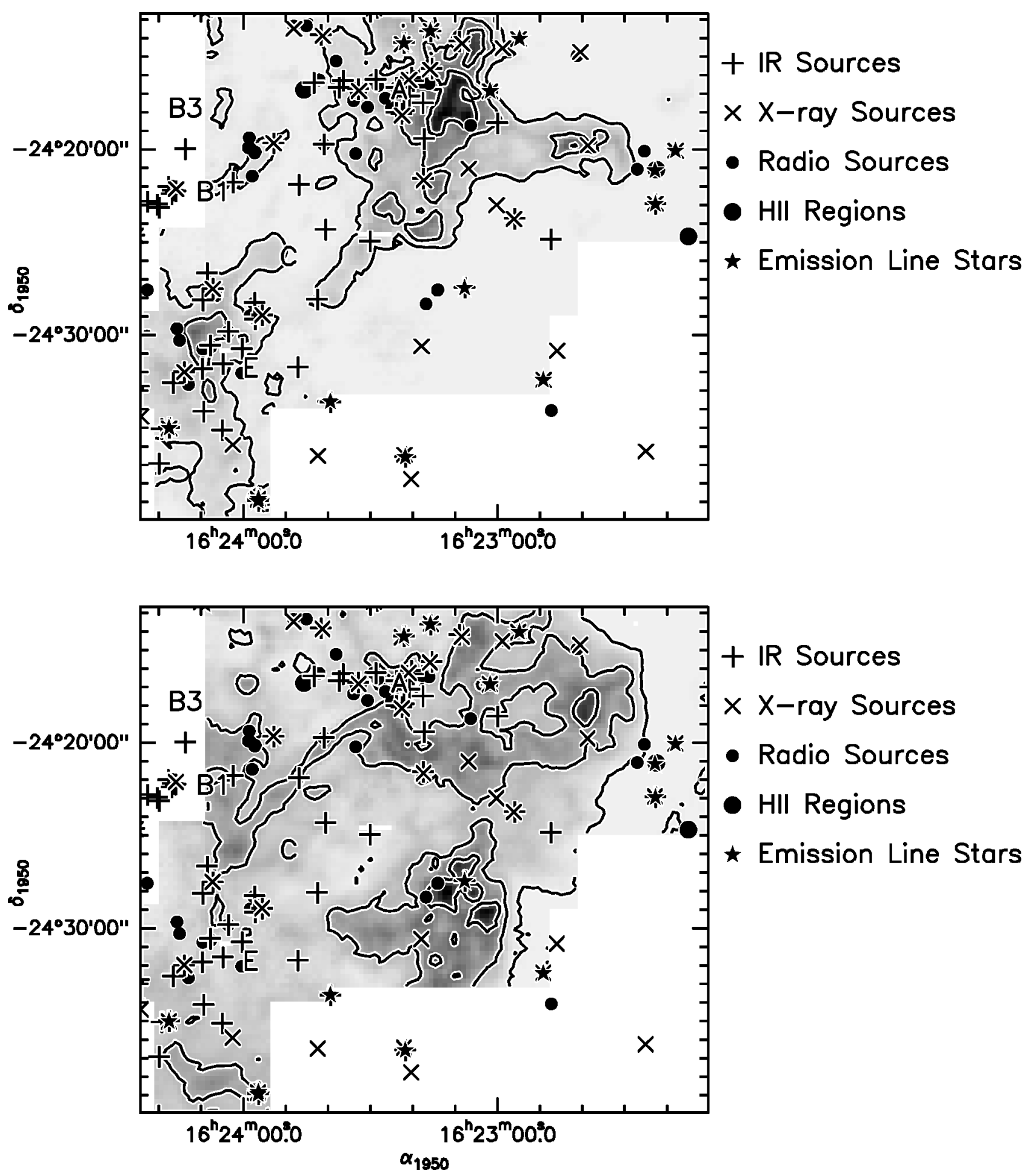

Fig. 3.-Locations of near-infrared, X-ray, radio sources, and emission-line stars associated with the $\rho$ Oph cloud. Sources marked as H II regions are bright compact radio sources from Brown \& Zuckerman (1975). Remaining radio sources are mostly T Tauri stars. Symbols are overlaid on top of images of the $\mathrm{CO}(2-1)$ integrated intensity in the velocity intervals $4-8 \mathrm{~km} \mathrm{~s}^{-1}$ and $0-4 \mathrm{~km} \mathrm{~s}^{-1}$ (upper and lower panels, respectively).

$-24^{\circ} 17^{\prime} 47^{\prime \prime}$; André, Ward-Thompson, \& Barsony 1990). Also, SR $4\left(16^{\mathrm{h}} 22^{\mathrm{m}} 54^{\mathrm{s}} 8,-24^{\circ} 14^{\prime} 01^{\prime \prime}\right)$ has been found to be the source of a string of $\mathrm{HH}$ objects (Wilking et al. 1997).

\subsection{Centroid Velocity Increments in the $\rho$ Ophiuchi Cloud}

A gray-scale map of the $\mathrm{CO}(2-1)$ centroid velocity in the $\rho$ Oph cloud is shown in the top panel of Figure 4. In the bottom panels we show PDFs of the centroid velocity increments computed for separations $\Delta=2,5,10$, and 20 pixels $\left(1\right.$ pixel $\left.=15^{\prime \prime}=0.5 \times \mathrm{FWHM}\right)$. As in Lis et al. (1996), all histograms presented here are normalized to a zero mean and a standard deviation of unity. A normalized Gaussian distribution is also shown as a dotted line in each panel. The magnitude of the non-Gaussian wings in the histograms of the centroid velocity increments decreases monotonically with the increasing separation $\Delta$. This evolution is similar to that in the simulation (see Fig. 6), although for the $\rho$ Oph histograms, the wings are somewhat more 

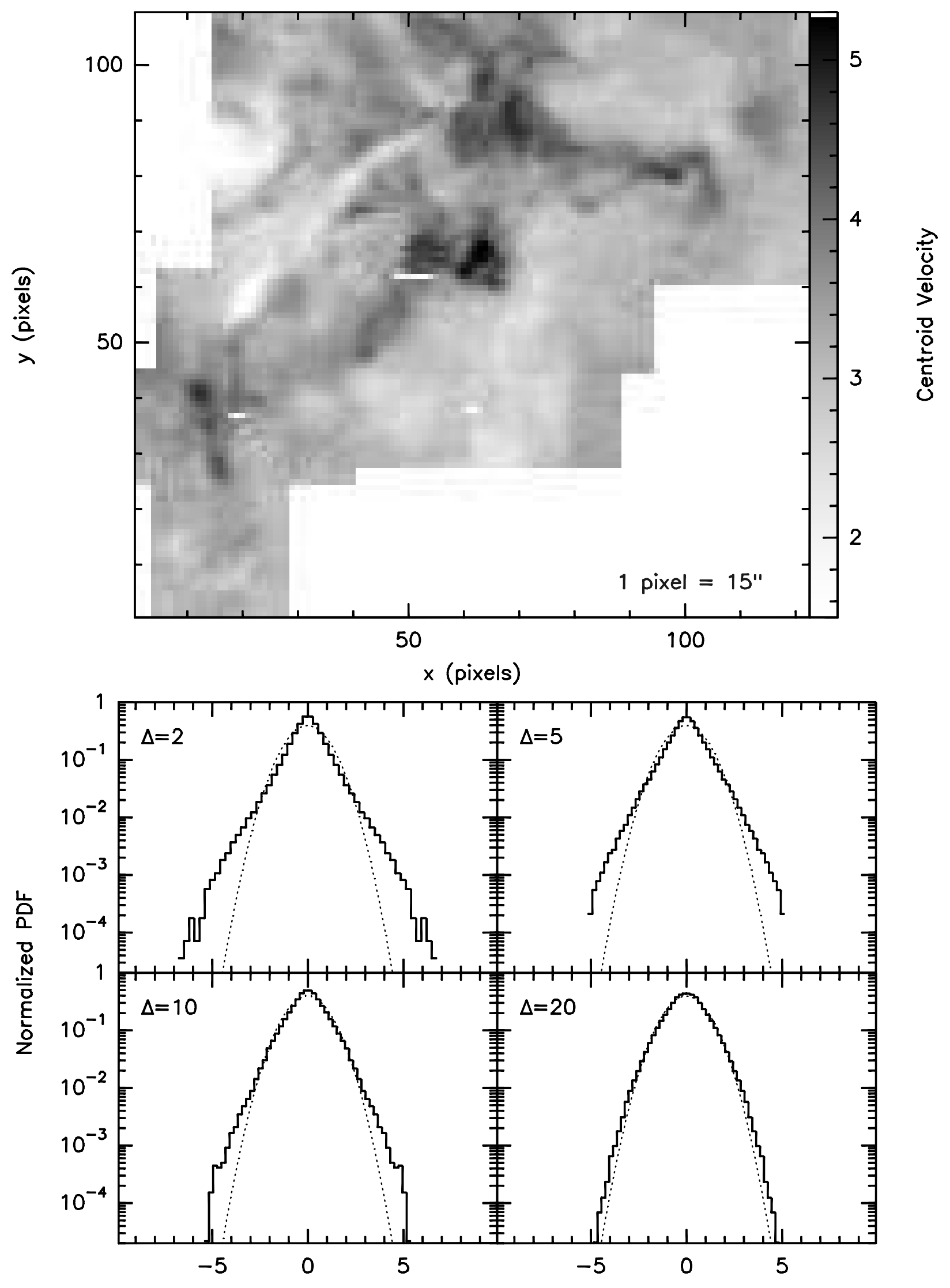

Normalized Centroid Velocity Increment

FIG. 4.-Top: Gray-scale map of the CO (2-1) line centroid velocity in the $\rho$ Oph cloud. Spatial coordinates are in pixels $\left(1\right.$ pixel equals $15^{\prime \prime}$, or one-half of the FWHM beam size). Bottom: Normalized histograms of centroid velocity increments in the $\rho$ Oph cloud for separations $\Delta=25,10$, and 20 pixels. 

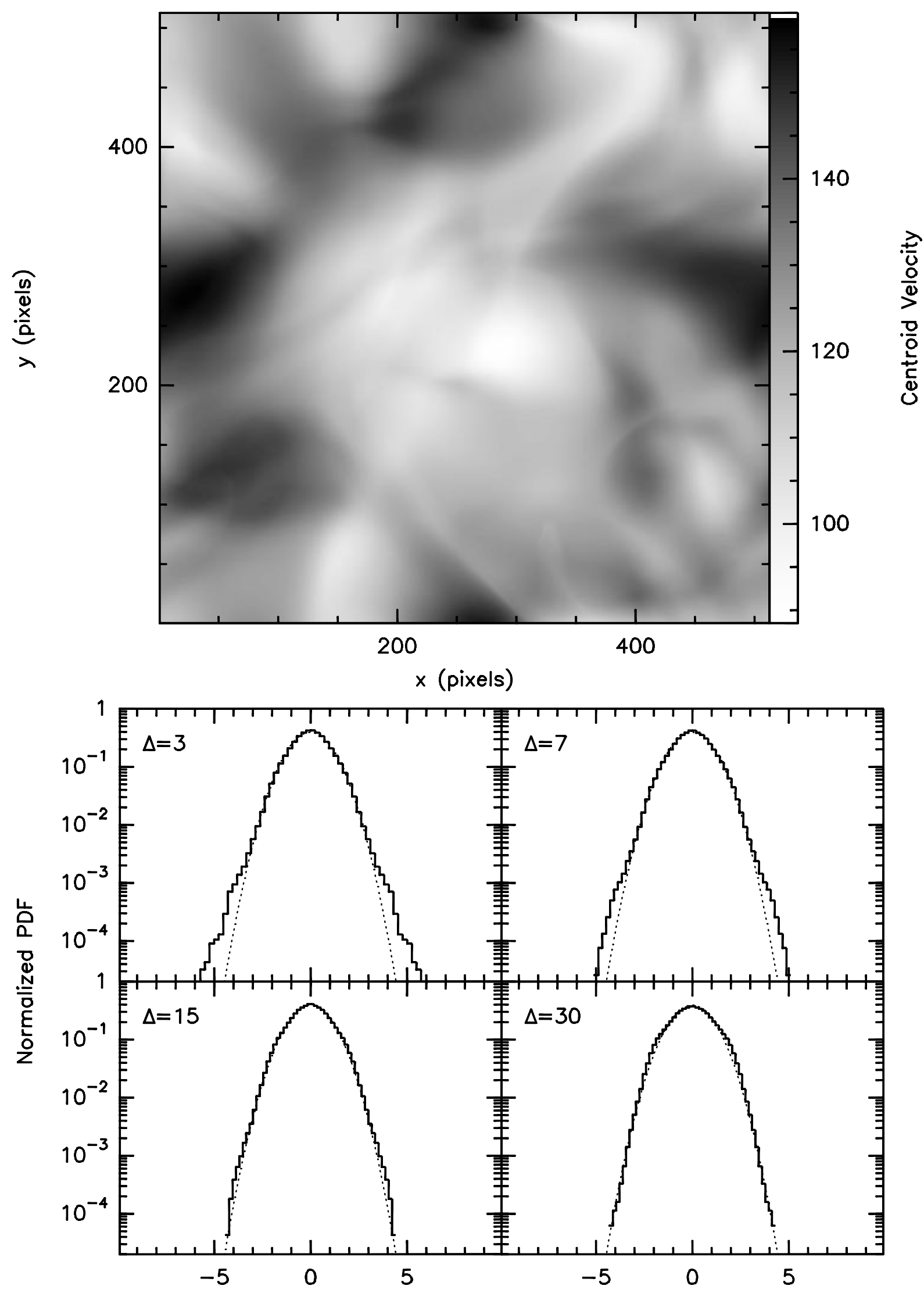

Normalized Centroid Velocity Increment

FIG. 5.-Map and histograms of the line centroid velocity increments for the first phase of the simulation $\left(t=0.5 \tau_{\mathrm{ac}}\right)$. The non-Gaussian wings are less pronounced compared to phase two (Fig. 6) for any value of $\Delta$. For $\Delta \gtrsim 15$, histograms are close to a Gaussian. 
pronounced at small separations, and the core of the histogram is more peaked.

Several morphological features can be identified in the centroid velocity map. The most prominent is a narrow low-velocity (blueshifted) filament running at $\sim 45^{\circ}$ position angle in the northeast quarter of the map. Emission associated with this filament can be seen at velocities between 0 and $2 \mathrm{~km} \mathrm{~s}^{-1}$ in the channel maps (Fig. 2). This feature is the blue lobe of the molecular outflow originating near VLA 1623 (André et al. 1990). A somewhat less prominent high-velocity (redshifted) filament can be seen to the southwest of the low-velocity filament in the centroid velocity map (Fig. 4). No feature corresponding to the highvelocity filament can be distinguished easily in the integrated intensity map. In addition, two compact regions with low and high velocities are seen on the opposite sides of the two narrow filaments. There is also an east-west filament in the northwest quarter of the map. All these features can be identified in the channel maps (Fig. 2).

\subsection{Time Evolution of the Centroid Velocity Increments in the Simulation and the Effects of Shocks}

As described in Falgarone et al. (1994), the time evolution of the simulation of Porter et al. (1994) is described as comprising three major phases. Their duration is conveniently expressed in terms of the acoustic time $\tau_{a c}=L_{0} / c_{S}$, which is the sound-crossing time of the energy-containing scale, $L_{0}=2 \pi / k_{0}$, where $c_{S}$ is the sound speed in the medium. The first short phase ends at $t \sim 0.3 \tau_{\mathrm{ac}}$, with the formation of first shocks. Shock interactions are the basic effects in the second supersonic phase where the vorticity generated by these interactions, as well as by vortex stretching, allows most of the kinetic energy to be transferred to solenoidal modes. The energy dissipation rate is thus considerably reduced, which explains the long duration of this phase, up to $t \sim 2.1 \tau_{\mathrm{ac}}$. In this phase and thereafter the flow has most of the properties of incompressible turbulence. The power spectrum for the solenoidal part of the flow (which contains more than $90 \%$ of the energy) is close to that predicted by Kolmogorov $\left[E(k) \sim k^{-5 / 3}\right]$. The PDF of the vorticity in this phase shows a conspicuous non-Gaussian tail, illustrative of the intermittency of the flow. The last, postsupersonic phase is that of the self-similar decay of the turbulent velocity spectrum.

Lis et al. (1996) studied centroid velocity increments in the second phase of the simulation, $t=1.2 \tau_{\mathrm{ac}}$, when the solenoidal small-scale modes have already gained a large fraction of the energy and almost all strong shocks have disappeared (Porter et al. 1994). To understand better the origin of the non-Gaussian wings in the histograms of centroid velocity increments, we have performed a similar analysis for the remaining epochs of the simulation. Results for the first phase, $t=0.5 \tau_{\mathrm{ac}}$, just after the shock formation, are shown in Figure 5. At this early time, the vorticity is very low, and small-scale disturbances have not yet developed. The velocity field is not yet characteristic of turbulence. The centroid velocity map for the second, supersonic phase of the simulation $\left(t=1.2 \tau_{\mathrm{ac}}\right)$ is shown in Figure 6 (top panel), along with the histograms of the centroid velocity increments (bottom panels). Finally, results for the third phase of self-similar decay of turbulence $\left(t=2.4 \tau_{\mathrm{ac}}\right)$ are shown in Figure 7.

In the first phase of the simulation (Fig. 5), non-Gaussian wings in the histograms of centroid velocity increments are seen only for small separations, $\Delta$. For $\Delta \gtrsim 15$, the histograms have essentially Gaussian shapes. As time progresses, the magnitude of the wings increases. The effect is most pronounced at larger separations $(\Delta \gtrsim 7)$; non-Gaussian wings are clearly present even for $\Delta=30$ in the second phase of the simulation (Fig. 6). The magnitude of the wings increases monotonically with decreasing $\Delta$ down to the numerical resolution of the simulation ( $\sim$ several pixels $)$. At even later times (Fig. 7), the magnitude of the wings increases further for large separations $(\Delta \gtrsim 15)$; however, the wings in the histograms for small separations are not very prominent. As discussed by Falgarone et al. (1994), in this late phase of the simulation most of the structure is at small scales, while large-scale structures have already disappeared. The line-of-sight averaging may thus be more effective in smearing out the most extreme events for small separations than at the earlier epochs of the simulation.

The time evolution of the non-Gaussian wings in the histograms of centroid velocity increments in the simulation (in particular the increase in the magnitude of the wings between phases one and two) is consistent with the evolution of vorticity in the flow, as described above. However, shocks may also have their signatures in the velocity field in the supersonic phase of the simulation. We can show, based on a simple geometrical argument, that planar shocks will have limited influence on the shapes of the histograms of centroid velocity increments. Lines of sight contributing to the non-Gaussian wings are associated with large centroid velocity shifts on small angular scales. In the case of a planar shock with the velocity in the plane of the sky (perpendicular to the line of sight), the shock front is well defined in the observations; however, there is no line-ofsight velocity jump, since the velocity of the shock is in the plane of the sky. Alternatively, for a shock with the velocity along the line of sight, there is a large line-of-sight velocity jump between the preshock and postshock gas, but we do not observe a well-defined shock front (the preshock and postshock gas overlap spatially on the sky). Therefore, in neither case will there be a strong contribution to the centroid velocity increments; however, the regions of interactions between shocks are likely to exhibit large velocity gradients over small angular scales. Therefore, we cannot exclude the possibility that shock interactions contribute to the non-Gaussian wings in the histograms of centroid velocity increments. Being able to identify shock interaction regions, however, is also interesting, since (as discussed by Porter et al. 1994) the vorticity is generated in shock interactions.

The increment method will also select boundaries of cloud fragments spatially superposed on the sky, which are of no interest to us and can only be identified by inspecting individual spectra.

\subsection{Distribution of Points Selected by Increments Method}

Lis et al. (1996) showed that the lines of sight contributing to the non-Gaussian wings of the PDFs of centroid velocity increments in the simulation trace a filamentary structure, which follows the distribution of the two vorticity components involving cross-derivatives of the line-of-sight component of the velocity field. The distributions of the lines of sight in the $\rho$ Oph map with centroid velocity increments greater than 3.5 for a separation $\Delta=2$ are shown in Figure 8. As in the simulation, the points are not randomly distributed but instead trace a number of coherent structures 

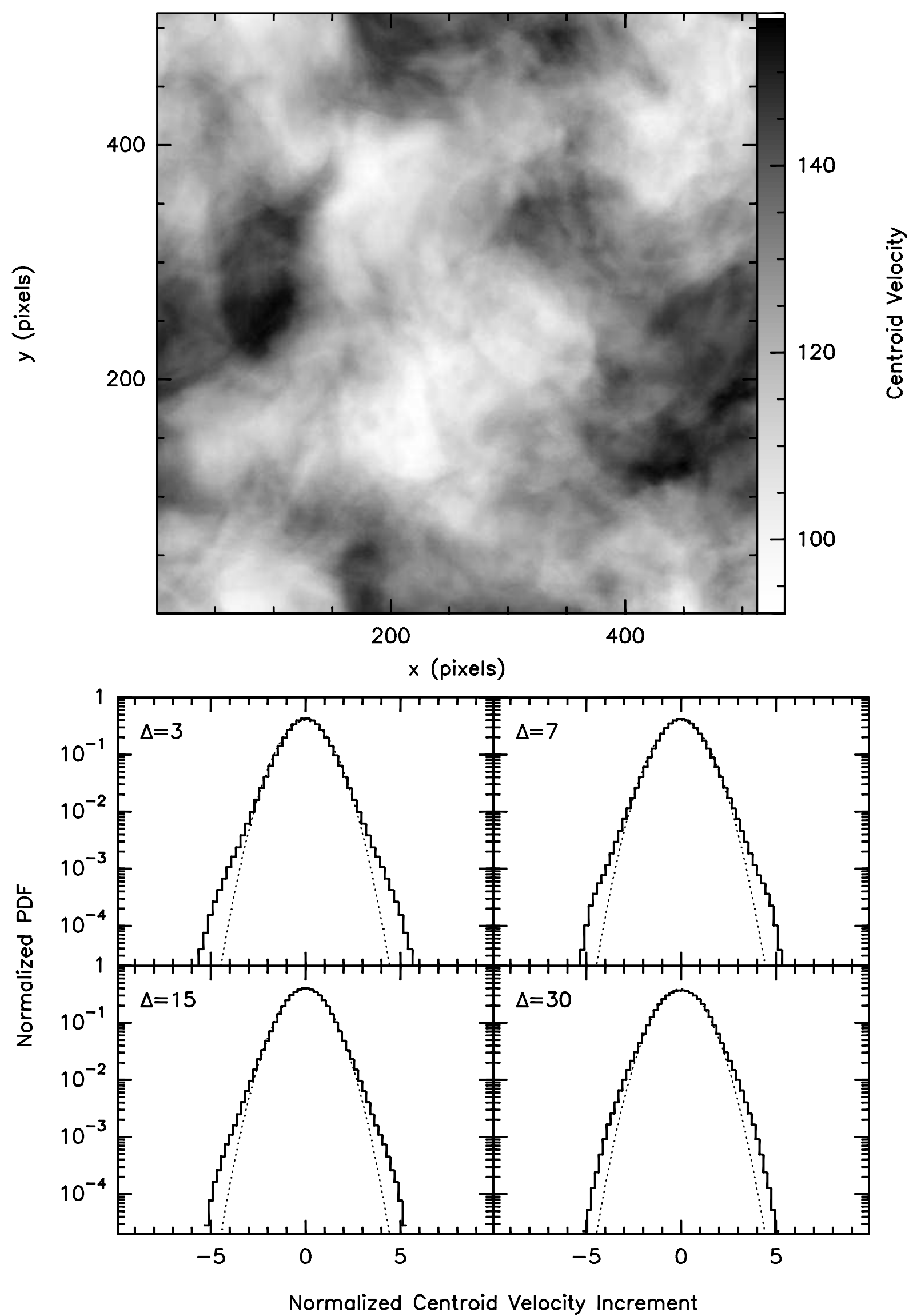

FIG. 6.-Top: Gray-scale map of the line centroid velocity for the second phase $\left(t=1.2 \tau_{\mathrm{ac}}\right)$ of the simulation of Porter et al. (1994). Note that the axes are reversed compared to Lis et al. (1996, Fig. 6). Bottom: Normalized histograms of centroid velocity increments computed for separations $\Delta=3$, 7, 15 , and 30 pixels. 

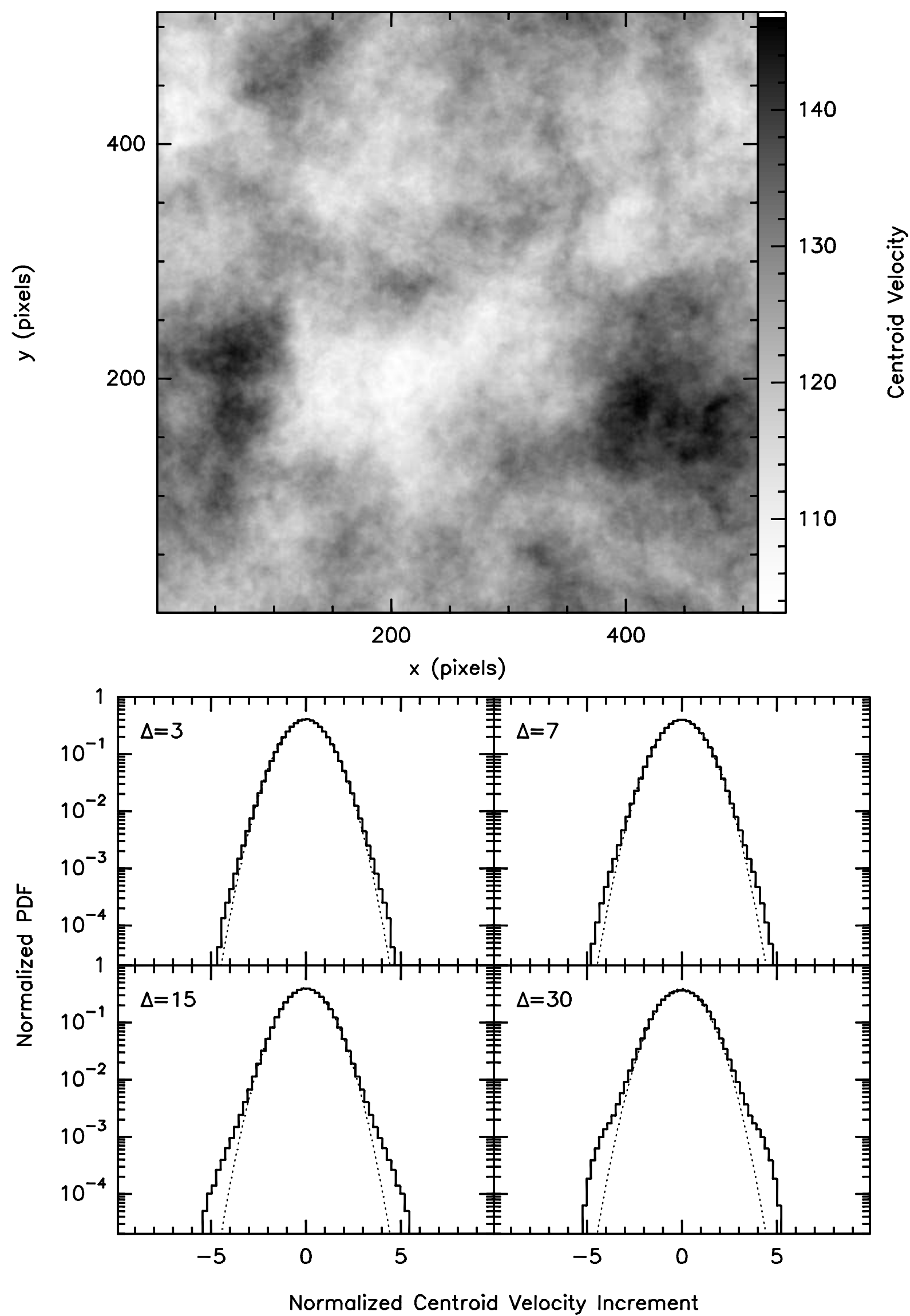

Fig. 7.-Map and histograms of the line centroid velocity increments for the third phase of the simulation $\left(t=2.4 \tau_{\text {ac }}\right)$. The non-Gaussian wings are still present for large separations $(\Delta \gtrsim 15)$. 


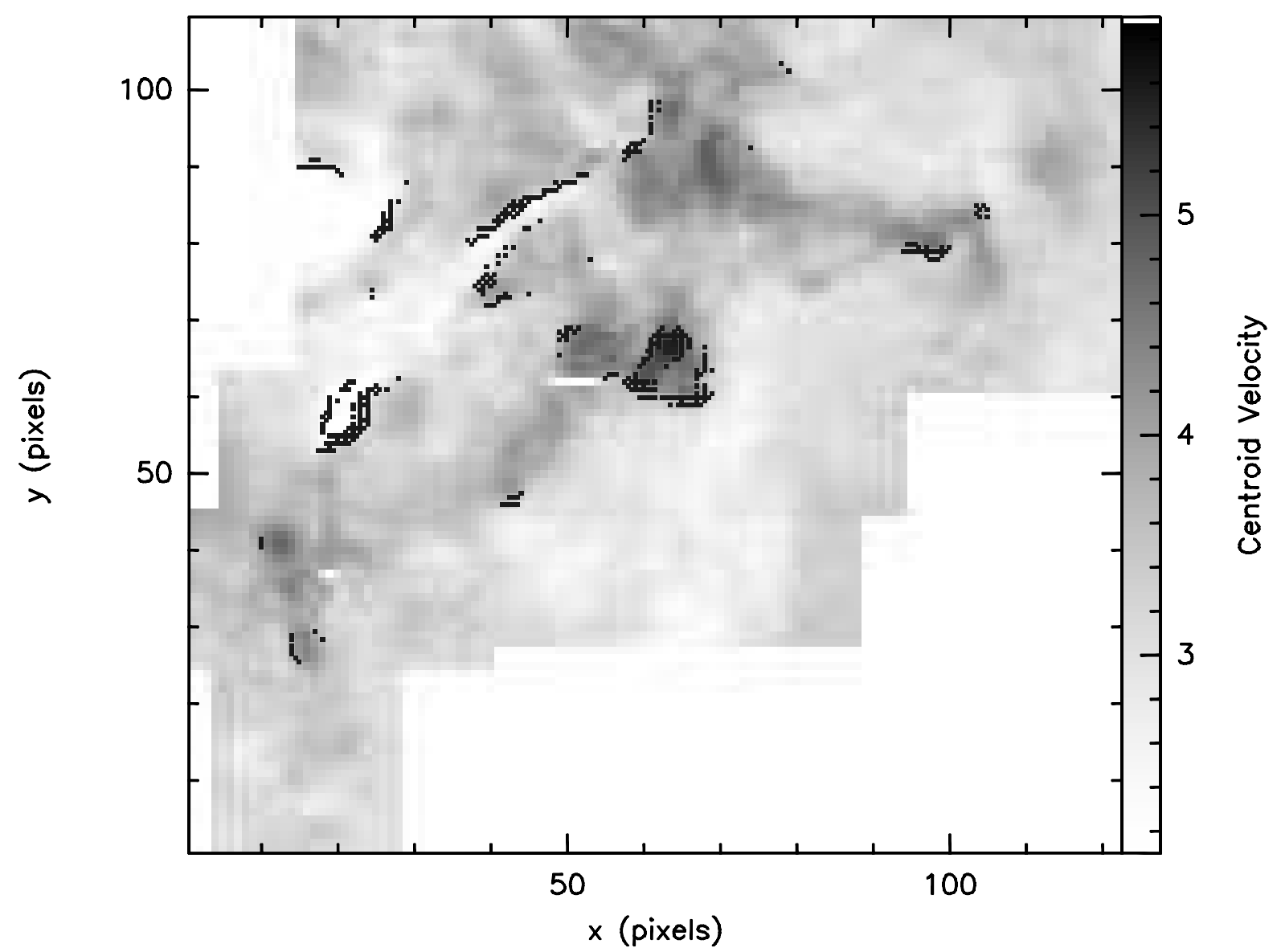

FIG. 8. -Distribution of the lines of sight in the $\rho$ Oph centroid velocity map with normalized centroid velocity increments greater than 3.5 for a separation of $\Delta=2$ pixels.

described in $\S 3.1$, namely the narrow filaments, high- and low-velocity compact sources, and the east-west filament.

\subsection{Optical Depth Effects}

The CO (2-1) lines in the $\rho$ Oph cloud have high optical depths. In an optically thick case, the line emission does not trace uniformly the velocity field along the line of sight, but instead it is sensitive primarily to the gas near the surface of the cloud (note that the optical depth in the line wings may still be low). Unless the turbulent velocity field in the surface layer is statistically different from that inside the cloud, we do not expect significant differences in the shape of histograms of centroid velocity increments for optically thick and thin lines. Lis et al. (1996) considered centroid velocities calculated with density weighting (appropriate for optically thin lines), as well as uniform weighting (more appropriate for optically thick lines originating in a clumpy, supersonic medium), and showed that the resulting histograms are similar.

To study the optical depth effects more quantitatively, including spatial overlap of optically thick cells, we computed simulated spectra for the second epoch of the simulation for different optical depths through the cloud assuming LTE conditions. We assumed that the optical depth through each cell of the data cube is proportional to the gas density in the cell and that the excitation temperature is uniform over the whole cube. Figure 9 shows a comparison of histograms of centroid velocity increments computed for an increment $\Delta=3$ for the second phase of the simulation, in optically thin and thick cases (average line center optical depths of 0.01 and 10, black and gray lines, respectively). As expected, the distributions corresponding to optically thin and thick cases are very similar, which indicates that optical depth effects do not influence significantly the shapes of the

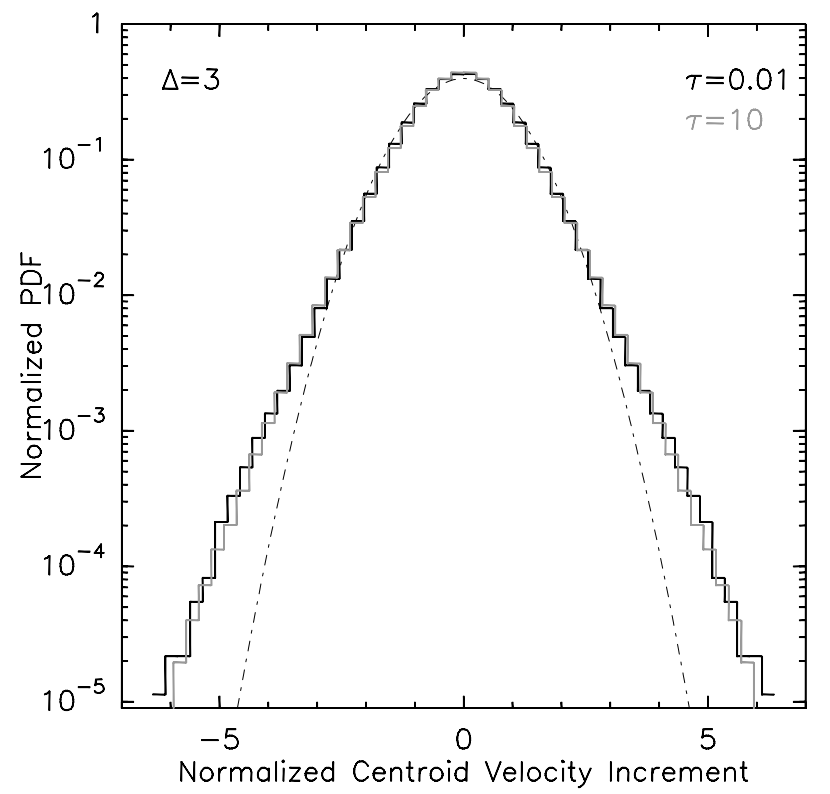

FIG. 9.-Comparison of histograms of centroid velocity increments for optically thin and thick lines. The histograms were computed for a separation of $\Delta=3$ for the second phase of the simulation. Black and gray lines correspond to mean line center optical depths of 0.01 and 10, respectively. 
histograms. From the point of view of statistical sampling of the molecular cloud velocity field, using optically thick lines is an advantage, since the emission is strong and it is relatively easy to collect a large enough data set for a meaningful statistical study. However, the distribution of points contributing to the wings of the histograms is different in the two cases. Since in the optically thick case the emission does not uniformly trace the gas along the line of sight, the increment method selects preferentially the regions with increased vorticity located closest to the observer. Highvorticity regions located along the line of sight deeper inside the cloud would largely remain undetected. Therefore, from the point of view of selecting lines of sight for comparative chemical and physical studies of turbulent regions with the bulk of the gas, the increment method is best applied to optically thin lines.

\section{SUMMARY}

The time evolution of the non-Gaussian wings in the histograms of centroid velocity increments in a compressible, but non-star-forming and nongravitating, turbulent simulation is consistent with the evolution of the vorticity in the flow. In particular, the magnitude of the wings increases between phase one and phase two of the simulation as shocks dissipate and the vorticity is generated; however, we cannot exclude the possibility that shock interactions contribute to the non-Gaussian wings present in the simulation.

Histograms of CO (2-1) centroid velocity increments in the $\rho$ Oph cloud are similar to those found in the simulation. This is consistent with the proposition that ISM velocity structure is vorticity dominated like that of the turbulent simulation; however, the region we have studied contains some active star formation, as indicated by the presence of infrared sources and molecular outflows. As a result, shocks may have more important effects on the velocity field structure and molecular line shapes in this region compared to the simulation. In particular, regions of shock interactions may contribute to the wings of the histograms of centroid velocity increments in the $\rho$ Oph cloud. Being able to identify shock interaction regions in the ISM is also important, however, since numerical simulations show that vorticity is generated in shock interactions.

We have demonstrated that the increment method can be successfully applied as a tool for studying the velocity field in interstellar molecular clouds. The same analysis now has to be applied to a carefully selected quiescent region without signs of ongoing star formation. The presence of nonGaussian wings in histograms of line centroid velocity increments in such a region would be a strong argument in favor of the turbulent nature of the velocity field in interstellar molecular clouds. Also, since the energy injected into the cloud by the embedded sources may play an important role in generating the turbulent cascade, a comparison of the histograms of centroid velocity increments in active and quiescent regions may help in understanding similarities and differences in the statistical properties of the velocity field between different regions in the ISM.

We thank D. H. Porter, A. Pouquet, and P. R. Woodward for providing us with the result of their hydrodynamic simulation, and E. Falgarone for helpful comments concerning the manuscript. This research has been supported by NSF grant AST 96-15025 to the Caltech Submillimeter Observatory, and by the JPL Supercomputing Project that is sponsored by JPL and the NASA Office of Space Science and Applications.

\section{REFERENCES}

Andre, P., Ward-Thompson, D., \& Barsony, M. 1993, ApJ, 406, 122

Anselmet, F., Gagne, Y., Hopfinger, E. J., \& Antonia, R. 1984, J. Fluid Mech., 140, 63

Barsony, M., Kenyon, S. J., Lada, E. L., \& Teuben, P. J. 1997, ApJS, 112, 109

Brown, R. L., \& Zuckerman, B. 1975, ApJ, 202, L125 Casanova, S., Montmerle, T., Feigelson, E. D., \& André, P. 1995, ApJ, 439,

de Geus, E. J., \& Burton, W. B. 1991, A\&A, 246, 559

de Geus, E. J., de Zeeuw, P. T., \& Lub, J. 1989, A\&A, 216, 44

Dubinski, J., Narayan, R., \& Phillips, T. G. 1994, ApJ, 448, 226

Elias, J. H. 1978, ApJ, 224, 453

Falgarone, E. 1997, in IAU Symp. 170, Twenty-Five Years of MillimeterWave Spectroscopy, ed. W. Latter et al. (Dordrecht: Kluwer), 119

Falgarone, E., Lis, D. C., Phillips, T. G., Pouquet, A., Porter, D. H., \& Woodward, P. R. 1994, ApJ, 436, 728

Falgarone, E., \& Phillips, T. G., 1990, ApJ, 359, 344

Falgarone, E., Phillips, T. G., \& Walker, C. W. 1991, ApJ, 378, 186

Gagne, Y. 1987, Thèse d'Etat, Univ. de Grenoble

Grasdalen, G. L., Strom, K. M., \& Strom, S. E. 1973, ApJ, 184, L33

Greene, T. P., \& Young, E. T. 1992, ApJ, 395, 516
Larson, R. B. 1981, MNRAS, 194, 809

Lis, D. C., Pety, J., Phillips, T. G., \& Falgarone, E. 1996, ApJ, 463, 623

Miesch, M. S., \& Scalo, J. M. 1995, ApJ, 450, L27

Montmerle, T., Koch-Miramond, L., Falgarone, E., \& Grindlay, J. E. 1983, ApJ, 269, 182

Myers, P. C. 1997, in IAU Symp. 170, Twenty-Five Years of Millimeter-

Wave Spectroscopy, ed. W. Latter et al. (Dordrecht: Kluwer), 137

Nozakura, T. 1990, MNRAS, 243, 543

Pfenniger, D., \& Combes, F. 1994, A\&A, 285, 94

Porter, D. H., Pouquet, A., \& Woodward, P. R. 1994, Phys. Fluids, 6, 2133

Scalo, J. M. 1987, in Interstellar Processes, ed. D. Hollenbach \& H. Thronson (Dordrecht: Reidel), 349

. 1990, in Physical Processes in Fragmentation and Star Formation, ed. R. Capuzzo-Dolcetta et al. (Dordrecht: Kluwer), 151

Strom, K. M., Kepner, J., \& Strom, S. E. 1995, ApJ, 438, 813

Vincent, A., \& Meneguzzi, M. 1991, J. Fluid Mech., 225, 1

Wilking, B. A., \& Lada, C. J. 1983, ApJ, 274, 698

Wilking, B. A., Lada, C. J., \& Young, E. T. 1989, ApJ, 340, 823

Wilking, B. A., Schwartz, R. D., Fanetti, T. M., \& Friel, E. D. 1997, PASP, 109,549

Zuckerman, B., \& Evans, N. J. 1974, ApJ, 192, L149 